\title{
Using Learner Group Profiles for Content Recommendation in Ubiquitous Environments
}

\author{
Luis Gustavo Ferreira, University of Vale do Rio dos Sinos, Brazil \\ Jorge Luis Victória Barbosa, University of Vale do Rio dos Sinos, Brazil \\ (iD) https://orcid.org/0000-0002-0358-2056 \\ João Carlos Gluz, IFRS - Federal Institute of Education, Brazil \\ Vítor Kehl Matter, University of Vale do Rio dos Sinos, Brazil \\ (iD) https://orcid.org/0000-0001-9422-0757 \\ Debora Nice Ferrari Barbosa, Feevale University, Brazil \\ iD https://orcid.org/0000-0001-8107-8675
}

\begin{abstract}
The application of ubiquitous technologies in the improvement of education strategies is called ubiquitous learning. This strategy amplifies the pedagogical potential of e-learning through a ubiquitous and contextualized perspective. On the other hand, a ubiquitous technological mediation in learning can also increase the isolation of learners and reduce the integration among colleagues. Strategies to encourage the group learning can minimize these possible side effects. In this sense, this article proposes UbiGroup, an agent-based model for ubiquitous recommendation of educational contents for groups of learners. UbiGroup aims to help teachers to search, select and distribute educational materials for groups. The model considers the group profile and the context where learners are. The recommendation for dynamic groups of learners through a consensus profile is the main scientific contribution of this research. The model was evaluated through simulated scenarios. The results were encouraging and show potential for implementing UbiGroup in real learning environments.
\end{abstract}

\section{KEYWORDS}

Context-Aware Computing, Groups, Learning Environment, Learning Objects, Profile Management, Recommendation, Ubiquitous Computing, Ubiquitous Learning

\section{INTRODUCTION}

The ever-increasing use of portable devices, such as smartphones and tablet PCs, has stimulated the adoption of mobile computing in different application areas. The user who carries portable devices can explore wireless communication technologies to access resources in anywhere and anytime. In

This article, originally published under IGI Global's copyright on October 1, 2020 will proceed with publication as an Open Access article starting on January 25, 2021 in the gold Open Access journal, International Journal of Information and Communication Technology Education (converted to gold Open Access January 1, 2021), and will be distributed under the terms of the Creative Commons Attribution License (http://creativecommons.org/licenses/by/4.0/) which permits unrestricted use, distribution, and production in any medium, provided the author of the original work and original publication source are properly credited. 
addition, the widespread use of location systems (Hightower, LaMarca, \& Smith, 2006), such as the GPS, has allowed a contextualized access to information (Dey, 2001). In this scenario, the ubiquitous computing initially introduced by Weiser (1991) and Satyanarayanan (2001) is becoming reality (Barbosa et al., 2015). The ubiquitous computing is a computational model that aims to pro-actively serve the needs of users, acting in an invisible way. The goal is to provide a continuous integration between technology and the environment, helping users in their daily tasks.

The application of mobile and ubiquitous computing in the improvement of learning strategies has created two research fronts called mobile learning and ubiquitous learning. Mobile learning (m-learning) (Saccol et al., 2011; Tatar, 2003; Klein et al., 2018) is fundamentally about increasing learners' capability to carry their own learning environment along with them. M-learning is the natural evolution of e-learning. The mobile computing has the potential to make learning even more accessible. In m-learning model, mobile computers are still not embedded in the learners' surrounding environment, and as they cannot seamlessly obtain information about contexts (Dey, 2001).

On the other hand, ubiquitous learning (Barbosa et al., 2011; Wagner et al., 2014; Rosa et al., 2015; Abech et al., 2016; Pimmer, Mateescu, \& Gröhbiel, 2016; Guabassi et al., 2018) refers to learning supported by the use of mobile and wireless communication technologies, sensors and location/tracking mechanisms (Barbosa et al., 2018), which work together to integrate learners with their environments. In addition, ubiquitous learning systems can involve the collaborative development of learning contents and learning processes, as well as, the use of social media for informal learning, communication and encouragement of participation (Marinagi, Skourlas, \& Belsis, 2013).

Ubiquitous learning systems connect virtual and real objects, people and events, in order to support a continuous, contextual and meaningful learning. While the learner is moving with mobile device, the system dynamically supports learning process by communicating with embedded computers in the environment. The essence of Ubiquitous Learning is to realize which information can be presented throughout the learners' daily tasks, in different forms and places, and to link this data with the learners' educational process. Technologies that support Ubiquitous Learning should provide these aspects through mechanisms that allow knowing learners' profiles, contexts involving them, and how learners relate to contexts.

Nowadays there are works about recommendation of educational material, which are becoming increasingly important due to the dissemination of ubiquitous learning. The web already provides a huge quantity of materials that can be useful for educational purposes. In this scenario, teachers not only need to examine whether this vast quantity of materials available falls in line with the syllabus but, ideally, also check if they comply to the learning profiles of students (Akbulut \& Cardak, 2012; Felder \& Silverman, 1988; Peterson, Rayner, \& Armstrong 2009 ) and to the teaching context where the learning is occurring (Barbosa et al., 2011; Rosa et al., 2015; Abech et al., 2016, Guabassi et at., 2018).

However, the management of student digital profiles and ubiquitous pedagogical contexts could make the already complex tasks of planning the educational activities and the organization of lessons, even more difficult to the teachers. They already devote considerable time to accomplish these tasks, seeking appropriate learning materials for the teaching plan. In addition, due to the wide range of available digital content, the teacher may require significant effort to find appropriate educational materials. This problem becomes more complex when one considers not only individual aspects of a profile, but collaborative aspects in learning groups. This motivates the development of computational tools to help teachers (and students) to explore the potential of Ubiquitous Learning and, mainly to support the selection and recommendation of content for groups.

This work assumes that educational contents are already catalogued as Learning Objects (LO) (IEEE-LTSC, 2002; Wiley, 2001). Of course, with this assumption we are not allowing the search and selection of any material from the web. However, this is not very restrictive because the systems need only the metadata to catalog LO for recommendation purposes. There is a crescent trend to use metadata standards like DublinCore (Kunze \& Baker, 2007), IEEE-LOM (IEEE-LTSC, 2002) 
and OBAA (Gluz \& Vicari, 2012) to catalogue information about materials available in the web, allowing more productive and effective searches. Other LO standardization issues that could be more restrictive, like packaging or accessibility, are not of concern of this work.

Making an integrated use of the information provided by LO metadata, the ubiquitous learning could become instrumental for the recommendation process, not only because it allows the easy distribution of customized materials directly to the learners on time and at the appropriate place, but because it allows the precise content distribution based on the context information.

With these considerations as the background, we propose UbiGroup as an agent-based model to provide ubiquitous content recommendation for groups of learners. The main contribution of this research is the strategy to recommend for groups. UbiGroup uses the learners' profiles to determine a consensus group profile, which is used to recommend learning objects. In addition, UbiGroup considers the dynamicity of groups and the context where learners are.

This article is organized in five sections. The next section discusses related work in the area of recommendation for groups. Third section describes the UbiGroup. The fourth presents a scenario used to evaluate the model. Finally, in the last section, we draw some conclusions and plans for future work.

\section{RELATED STUDIES}

We considered as related works only models that contain mechanisms to recommend content for groups of users. The search was not restricted to works that recommend learning content because few articles were found with this focus. Table 1 presents a comparison among related works considering five aspects considered strategic in this research.

Boratto et al. (2010) proposed a model for automatic identification of user groups. The work created the ImprovedGRA recommendation algorithm for groups using the technique of collaborative filtering. For group identification, the model uses individual recommendations, combined with users' preferences and uses them as input to the clustering algorithm. The recommendation algorithm generates predictions from the classification matrix used to identify the groups and, then, adds the predictions of the items not rated by users.

Kim et al. (2010) proposed a recommendation procedure for groups in on-line communities, which is called GRec_OC. The procedure works by initially generating a set of recommendations for the group using the method of collaborative filtering. Soon after, the similarity between the profiles of the group is calculated and the determination of neighboring groups with greatest similarity is made. For each neighbor group a set of recommendations based on items most frequently purchased is selected. The final step consists of a filtering method to reduce the dissatisfaction of individual group members.

Christensen and Schiaffino (2011) described the development of two recommender systems for groups of people: jMusicGroupRecommender and jMoviesGroupRecommender. These systems recommend, respectively, music and movies. They were developed based on the framework GroupRecommendation. This work proposes two approaches for generating recommendations for groups: merging the individual recommendations made by aggregating the individual ratings of each item evaluated and developing a model of group preferences.

Webber and Lima (2012) considered that group work is an important resource for teachers to promote collaborative learning. So, they empirically studied one of the most important issues of this research field, namely, if automatic mechanisms for group formations can actually be able to form consistent and successful groups for education purposes. The experiment presented in the article shows that automatic techniques can produce results consistent with regard to the formation of groups.

Kardan and Ebrahimi (2013) proposed a hybrid recommender system. The similarity of users is identified based on implicit information about their interactions and web navigation. After, association rules are used to improve the collaborative filtering process. The content-based filtering process uses semantic techniques to identify relevant posts and automatically infers the formation of groups, uses 
rules to select the recommended collaborative material, and applies semantic techniques to filter the returned content.

Zapata et al. (2015) proposed a collaborative methodology focused on learners' groups for searching, selecting and rating learning objects. This methodology is an extension of DELPHOS which is a framework to assist users in the individual personalized search for learning objects in repositories. To group support, the authors included the creation and management of groups of users, the realization of collaborative activities, and the recommendation of the most interesting learning objects to these groups. A meta-learning approach is also proposed in order to help the mediator of a group to select the best rating aggregation method depending on the rating of previous similar groups.

Table 1. Related works comparison

\begin{tabular}{|c|c|c|c|c|c|c|}
\hline Aspects/Works & $\begin{array}{l}\text { Boratto et } \\
\text { al. (2010) }\end{array}$ & $\begin{array}{l}\text { Kim et } \\
\text { al (2010) }\end{array}$ & $\begin{array}{l}\text { Christensen and } \\
\text { Schiaffino (2011) }\end{array}$ & $\begin{array}{l}\text { Webber and } \\
\text { Lima (2012) }\end{array}$ & $\begin{array}{l}\text { Kardan and } \\
\text { Ebrahimi } \\
(\mathbf{2 0 1 3})\end{array}$ & $\begin{array}{l}\text { Zapata et } \\
\text { al. (2015) }\end{array}$ \\
\hline $\begin{array}{l}\text { 1. It uses a multiagent } \\
\text { system }\end{array}$ & No & No & No & No & No & Yes \\
\hline 2. It focus on learning & No & No & No & Yes & No & Yes \\
\hline $\begin{array}{l}\text { 3. It automatically infers } \\
\text { the formation of groups }\end{array}$ & No & No & No & No & No & Yes \\
\hline $\begin{array}{l}\text { 4. It uses rules to select } \\
\text { the recommended } \\
\text { content }\end{array}$ & Yes & Yes & Yes & Yes & Yes & Yes \\
\hline $\begin{array}{l}\text { 5. It applies semantic } \\
\text { techniques to filter the } \\
\text { content }\end{array}$ & No & No & Yes & Yes & Yes & Yes \\
\hline
\end{tabular}

Boratto et al. (2010) and Kim et al. (2010) proposed similar techniques to create groups and to content recommendation. The proposed systems do not focus on learning recommendation, as well as they do not use ubiquitous information to recommend content.

The works of Webber and Lima (2012), Kardan and Ebrahimi (2013) and Zapata et al. (2015) are the works most similar to UbiGroup. They automatically infer the formation of groups and use association rules to improve the collaborative filtering process. In addition, these proposals apply semantic techniques to identify relevant content.

UbiGroup also automatically infers the formation of groups, uses rules to select the recommended collaborative material, and applies semantic techniques to filter the returned content. However, there are two main differences, which are the contributions of UbiGroup. First, the techniques used to dynamically form groups and select materials are generalized to work with ubiquitous information of learners. The second difference relates to the semantic approach to filter results. Unlike the dictionary techniques used by Kardan and Ebrahimi (2013), and the framework elements explored by Zapata et al. (2015), UbiGroup uses a full ontological approach to handle the semantics of the filtered contents (learning objects). UbiGroup focuses on learning such as Webber and Lima (2012) and Zapata et al. (2015). In addition, UbiGroup and Zapata et al. (2015) use multiagent systems, but Zapata et al. (2015) does not consider ubiquitous information for reasoning.

UbiGroup was designed to work based on contexts and portable devices. The model uses context information to dynamically create groups of learners, and then use this information to select appropriate educational material. UbiGroup is dedicated to support educational environments using ontology to 
recommendation support. All information management focuses on learning support, including the profiles, contexts and learning objects.

\section{THE ARCHITECTURE OF UBIGROUP}

Figure 1 shows the architecture of the proposed model. UbiGroup is a multiagent system (Wooldridge, 2009) composed by five pedagogical agents (Giraffa \& Vicari, 1998; Vicari et al., 2008). These agents work autonomously and cooperatively in a distributed environment formed by mobile devices and computer servers. The overall architecture and all of its individual agents were designed using software engineering methods focused on developing pedagogical agents for tutoring systems (Vicari \& Gluz, 2007).

Figure 1. Agent-based architecture of UbiGroup model

\section{UbiGroup}

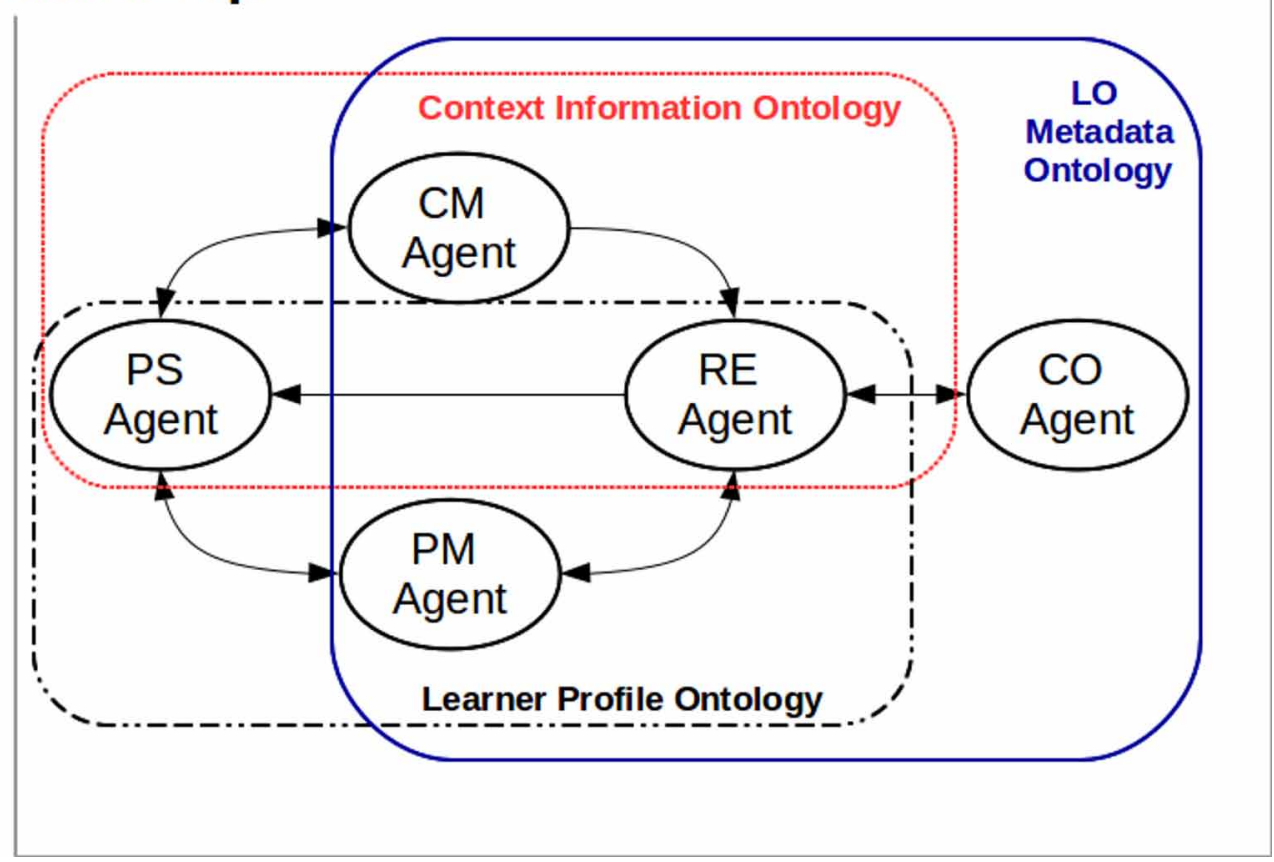

The Pedagogical Support agent (PS) obtains information from learners and informs them about new recommendations. The Profile Management agent (PM) keeps learner profiles updated and generates the similarity degree between these profiles. The Context Management agent (CM) manages the contexts in the ubiquitous environment. The Recommender agent (RE) maintains the base of recommendation rules. Finally, the Communicator agent (CO) performs communication with the repository of LO. Besides the requirements of ubiquitous computing and recommendation systems, there are other important requirements that impacted on the design of the model: a) groups of learners can be formed dynamically, namely, they can be created and dissolved at any time; b) information about learning materials are represented in the form of LO metadata; c) information about learner profiles and contexts are also provided in the form of metadata. 
All metadata about learner profiles, teaching contexts and learning materials were represented in ontologies specified in OWL language (W3C, 2012). In this sense, UbiGroup followed the principles stablished in previous works (Gluz \& Vicari, 2012; Gluz, Vicari \& Passerino, 2012). OWL data properties (W3C, 2012) were used to represent these metadata, allowing us to use a previously developed OWL/RDF semantic base. This base can be used to solve SPARQL (W3C, 2013) queries over inferred ontologies (Gluz \& Silva, 2014) to store, locate and retrieve the metadata necessary for the recommendation process. The specific operational knowledge about how to identify and recommend materials was represented in the form of recommendation rules, which are analogous to the production rules used in expert systems (Russel \& Norvig, 2010). In UbiGroup, these rules map profiles, contexts and LO metadata information to recommendation filters. The following subsections detail the UbiGroup architecture.

\section{Pedagogical Support (PS) Agent}

The PS agent is the interface of UbiGroup with learners, being the only agent that runs on the mobile device. While is executing, this agent sends the updated information of profiles to PM agent and sends the updated location to CM agent. The PS also calls the PM agent for the validation of the learner's access credentials.

The main role of the PS agent is to monitor the movements of the learner and send this information to the $\mathrm{CM}$ agent. If the $\mathrm{CM}$ identifies that the learner is in a learning context, it informs the PM agent, which in turn, notifies the learner that he or she has entered a particular context.

During the recommendation process, the responsibility of the PS is to notify learners about recommendations for them. The agent also presents the recommended materials to learners. After viewing a material, the learner is asked to rate the recommendation indicating whether it was satisfactory or not.

\section{Profile Management (PM) Agent}

The PM agent keeps updated the profiles of learners. This agent also works with the CM agent to monitor the access of learners to contexts. The PM retrieves the credentials from the learner profile and sends these credentials to the CM, which in turn keeps track of which context the learner is.

The learner profile is represented by an ontological model specified in OWL (W3C, 2012). The classes and properties of this ontology were defined based on the PAPI standard for learner metadata (PAPI, 2002) (see Figure 2).

Profile information has six categories: contact, portfolio, grade, relation, preference and security. Personal information about the learner was collected in contact category. The portfolio category groups information about the works produced by the learner. The relation category collects information about the relationships with other learners. The grade category contains the grade evaluations of the learner. Preference category groups the preferences of the learner and security category indicates the access credentials.

The profile metadata were represented by OWL data properties directly associated with the individual (instance) representing the profile of a learner in the ontology. Thus, assuming that $p 1$ represents the profile of learner A, the identification and name of the learner would be represented by the following RDF triples:

\section{p1 learnerIDIs "1" \\ p1 nameIs "Student A"}

The PM agent also calculates the similarity degree between profiles of learners. The estimation of the similarity is based on values of Preference, Grade and Portfolio properties (see Figure 2). Before calculating the similarity between two profiles, all preference, grade and portfolio properties 


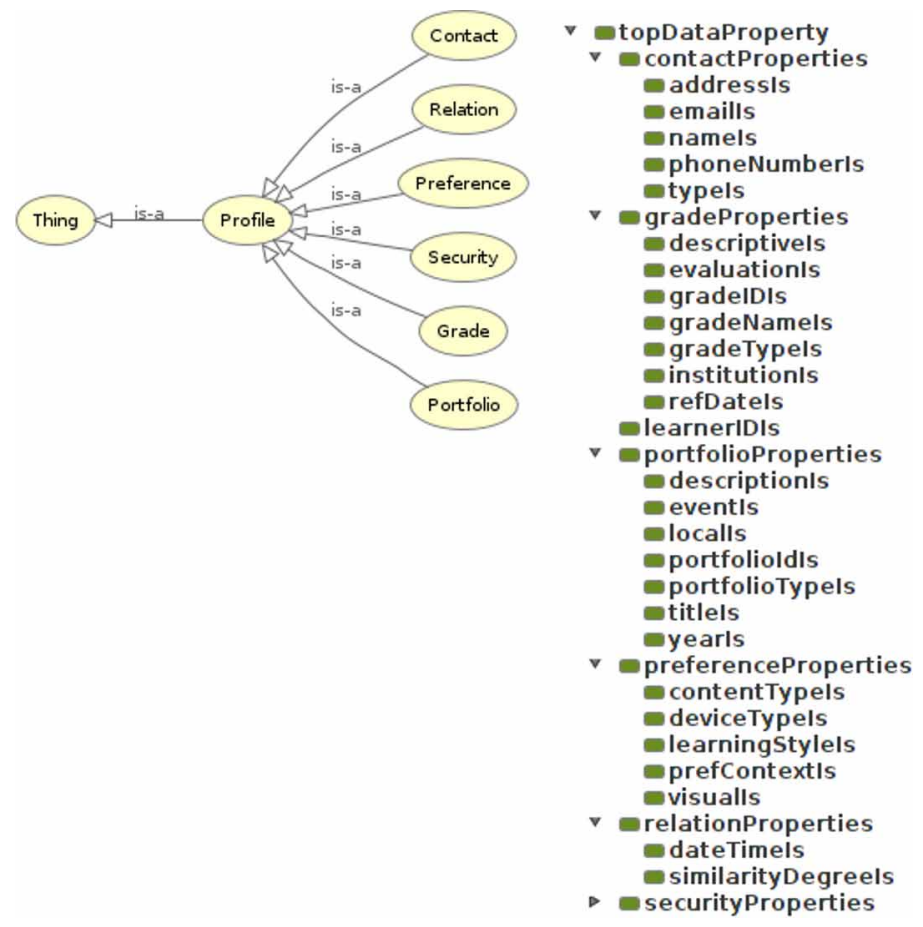

that coincide in both profiles (learners have the same property, but not necessarily the same value for the property) are selected to form a set of $\mathrm{n}$ item values, which is used in the comparison. Equation 1 shows how the degree of similarity is calculated based on this set of values:

$S\left(S P_{1}, S P_{2}\right)=\frac{\sum_{i=1}^{n}\left(S P_{1}, \text { item Value }_{i}, S P A_{2}, \text { item Value }_{i}\right)}{n}$

The similarity (S) between the profiles of two learners (SP1, SP2) is equal to the sum of the Value of Equivalence (VE) for each preference, grade and portfolio item stored on profiles of both learners, divided by the total number of items evaluated (n). The individual VE between each two items is estimated by the editDistance algorithm (Levenshtein, 1966). This algorithm compares two strings and determines the editing distance, which is the minimum number of operations needed to transform one string into another. With this distance is possible to calculate the similarity degree, dividing the number of operations (obtained by the algorithm) by the maximum number of possible operations. Note that to use the editDistance algorithm, we needed to set the range of all OWL data properties used in the learner profile ontology to the string data-type.

\section{Context Management (CM) Agent}

The CM agent maintains the base of contexts used for teaching purposes and informs the RE agent about changes regarding the entry or exit of learners in some context registered in the base. The context information used by CM is represented in an OWL ontology. The classes and properties of this ontology were based on the definitions proposed by Dey (2001). Figure 3 shows the main classes and data properties of the context ontology. 
Figure 3. High-level classes and properties of context ontology

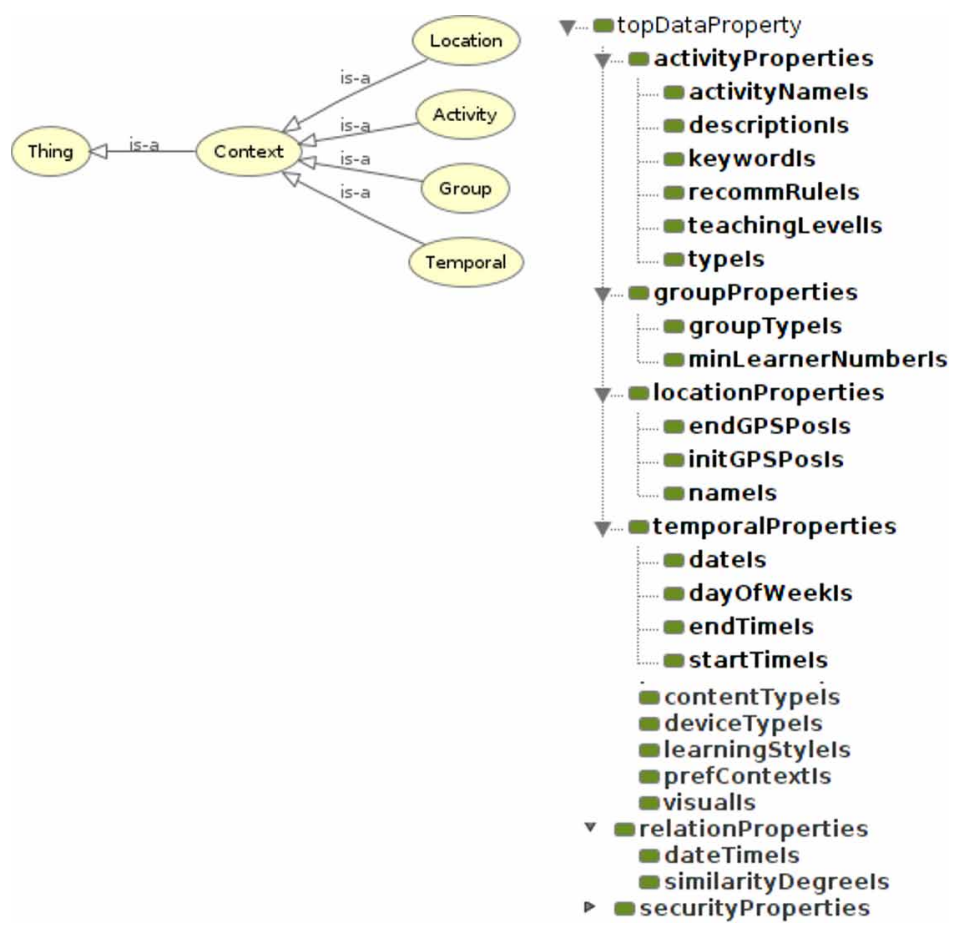

The context information is organized in four categories: location, activity, temporal and group. Location category collects geographical information about the context. The activity category collects information about activities that can occur in some context. Temporal category indicates the temporal limits for the context. The group category collects information related to the formation of groups of learners in the context. Following the same structure used in the profile ontology (Figure 2) and in the LO metadata ontology, specific metadata information about the context are represented by data properties associated with the ontology individual (instance), which uniquely identifies the context.

The CM maintains all contexts that are active, which are those having at least one learner. This, however, does not imply that the context is already able to receive content recommendations. This will occur only when this particular context meets the requirements in temporal and group categories. It is the CM agent that checks if these requirements were satisfied. Whenever a learner enters or leaves a context, the $\mathrm{CM}$ informs the RE agent about this movement, because this is one of the triggers to start the recommendation process.

\section{Recommender (RE) Agent}

The RE agent manages the recommendation process. It keeps the rules to select new recommendations, and performs the steps necessary to prepare, execute and forward the recommendation to learners.

A recommendation rule is formed by a set of mappings between profiles, learning contexts and LO metadata. From these mappings, it is possible to extract production rules to generate a set of filters that will select LO through metadata. The mappings on the recommendation rule correlate information contained in the profile, or learning context, with information appearing in LO metadata. All information that may appear in these rules is represented according with the corresponding OWL ontology. Learner profile and context information are represented by classes and properties of 
ontologies shown, respectively, in Figure 2, and Figure 3. The LO metadata used the ontology defined by Gluz and Vicari (2012), which covers all IEEE-LOM metadata (IEEE-LTSC, 2002).

Each mapping is formed by three items: the learner profile/context information, the corresponding LO metadata, and the value that should appear as the content of previous items. In all ontologies used by UbiGroup, the information is organized in the same way: (a) the class, which defines the LO metadata group, or the category of learner profile/context information; (b) the OWL object/ data property, which defines the LO metadata or learner profile/context attribute. Table 2 shows an example of a mapping from learner profile information to LO metadata. In this example, the type of the profile is mapped to the intended type of end user of the LO. Preferential content formats and digital devices are also mapped to specific technical LO metadata that can be used to select materials with these characteristics.

Table 2. Example of mappings between learner profile information and LO metadata

\begin{tabular}{|l|l|l|l|l|}
\hline \multicolumn{1}{|c|}{ Learner Profile } & \multicolumn{2}{c|}{ LO Metadata } & \multirow{2}{*}{ Value } \\
\hline \multicolumn{1}{|c|}{ Class } & \multicolumn{1}{|c|}{ Property } & \multicolumn{1}{c|}{ Class } & \multicolumn{1}{c|}{ Property } & \\
\hline Contact & typeIs & Educational & intendedEndUserRoleIs & "learner" \\
\hline Preference & contentFormatIs & Technical & formatIs & "video" \\
\hline Preference & deviceTypeIs & Technical & supportedPlatformIs & "mobile" \\
\hline
\end{tabular}

Table 3 shows an example of mappings from context information to LO metadata. This example shows how to use keywords about activities that will occur in the context, to select appropriate educational materials, looking in the title, description or keyword in the LO metadata.

Table 3. Example of mappings between context information and LO metadata

\begin{tabular}{|l|l|l|l|l|}
\hline \multicolumn{2}{|c|}{ Context Information } & \multicolumn{2}{c|}{ LO Metadata } & \multirow{2}{*}{ Value } \\
\cline { 1 - 3 } Class & \multicolumn{1}{|c|}{ Property } & \multicolumn{1}{|c|}{ Class } & \multicolumn{1}{c|}{ Property } & \\
\hline Activity & keywordIs & General & TitleIs & "java" or "programming language" \\
\hline Activity & keywordIs & General & descriptionIn & "java" or "programming language" \\
\hline Activity & keywordIs & General & KeywordIs & "java" or "programming language" \\
\hline
\end{tabular}

The mappings of recommendation rules provide the information that the $R E$ agent needs to generate metadata filters that will be used to select $\mathrm{LO}$ to a context and profile. These filters are sent to the $C O$ agent, which in turn will select the LO corresponding to the filters informed in the message. The recommendation rules used by the $R E$ agent can be managed through a web interface (see Figure 4, the interfaces are in Portuguese).

The recommendation process is initiated when a context is ready to receive a recommendation. For this, it is necessary that the temporal and group conditions for this context are fully satisfied. When this occurs, the selection of material to be recommended starts. The process is conducted by RE agent in three steps: (1) identification of the most representative profile for the group (consensus profile), (2) the selection of recommendations for this profile, and (3) classification of selected items based on reviews from group members. 
Figure 4. Web interface to manage recommendation rules (in Portuguese)

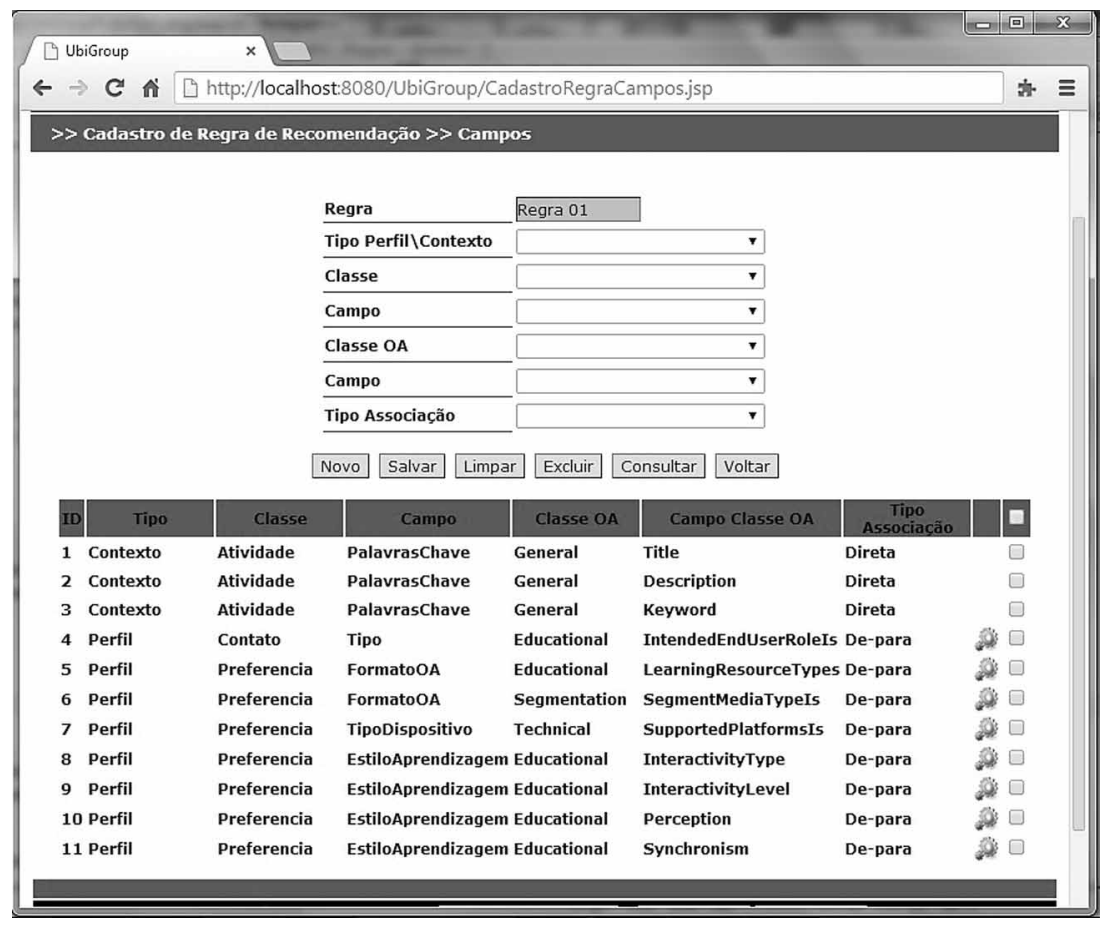

The first step starts with the analysis of the profiles of the learners in the context. This analysis identifies which is the most representative profile for the group using the degree of similarity between the profiles (see Equation (1)). This profile is used as the basis to select the LO. The profile that has the highest average similarity among the group members will be chosen as the representative profile. To identify this profile, firstly the RE agent requests the PM agent for calculating the similarity of each profile in the context with all other profiles in the same context. This analysis results in a similarity matrix (see Table 6 in advance, as an example). Thereafter, the RE searches profiles that have similarity greater than $90 \%$ with all other profiles. If no profile is found, the RE follows successively reducing in $10 \%$ the similarity threshold until be found at least one profile that meets to a minimum average value.

The second step begins with generating recommendations for the representative profile. Based on the profile, context and recommendation rules contained in the context, the $R E$ agent builds a set of LO filters, each one specified as a relational and logical expression over LO metadata. Then, these filters are sent to the $\mathrm{CO}$ agent. This agent transforms the filters in appropriate queries for the LO repositories registered in the system and returns to the RE, the lists of LO that satisfy the queries. After determining the recommendations to the representative profile, these recommendations are unified into a single list with the LO to be recommended for learners.

In the final step, the RE agent classifies the list, considering the assessments made by the group members. So, the best evaluated items will be the first to be recommended to the group. The RE agent selects and sends the learnings objects to PS agent.

\section{Communicator (CO) Agent}

The $\mathrm{CO}$ agent implements communication with $\mathrm{LO}$ repositories. This agent receives a request from the RE agent containing a set of search filters and transforms this data into a query compatible 
with the LO repositories. Then the $\mathrm{CO}$ accesses the repositories to obtain the information of LO. Communication with the repositories occurs through a web services interface. Finally, the list of LO returned by the repository is forwarded to the RE agent.

Currently, the CO agent works only with semantic repositories (Gluz, Silva \& Vicari, 2014) which support SPARQL queries over IEEE-LOM/OBAA metadata represented in OWL ontologies (Gluz \& Vicari, 2012). Figure 5 shows the SPARQL query corresponding to the rule mappings shown in Table 2.

\section{Figure 5. An example of SPARQL query generated by $\mathrm{CO}$ agent}

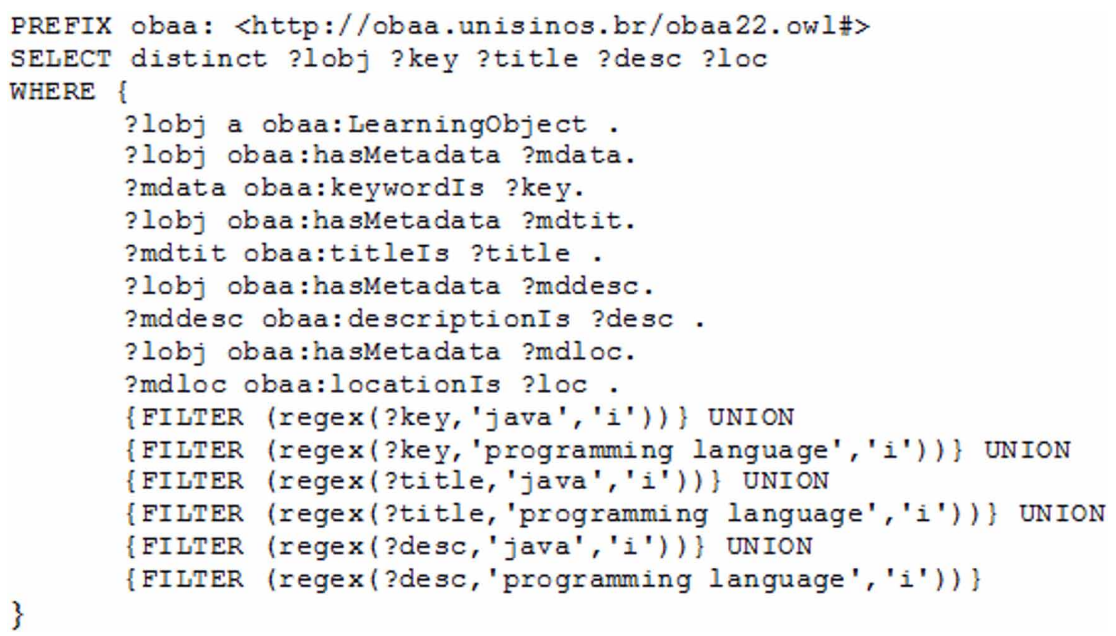

\section{IMPLEMENTATION ASPECTS}

UML was used for specifying the UbiGroup architecture. This modeling language has been considered suitable for the specification of the overall architecture of multiagent systems and their individual agents (Bauer \& Odell, 2005; Guedes \& Vicari, 2010).

The prototype implements a basic set of features for each agent, which were considered strategic to conduct the evaluation. The PS agent was developed for Android devices with the Android SDK support. Server-side agents (PM, CM, RE and CO) were developed in Java with support of Tomcat server for the web interface, REST/Jersey framework for web services interface, Jena library for handling OWL ontologies and SPARQL queries. The prototype used a semantic LO repository that is capable of dealing with SPARQL queries to search LO metadata. The metadata information retrieved is represented in XML.

\section{EVALUATION ASPECTS}

The evaluation methodology was based on a strategy of validation by scenarios. This approach was used in classical works to evaluate context-aware systems (Dey, 2001) and ubiquitous systems (Satyanarayanan, 2001). Most recently, this strategy has been applied to evaluate the use of ubiquitous computing in various application areas such as Health (Vianna \& Barbosa, 2014), Commerce (Barbosa et al., 2016), Accessibility (Barbosa et al., 2018), Competences Management (Rosa et al., 2015) and Learning (Barbosa et al., 2011; Wagner et al., 2014). 
Several scenarios were created to evaluate the UbiGroup. In this article, we discuss a specific scenario that evaluates the most significant features of the model. The scenario assumes a teacher in an undergraduate course in "Algorithms and Programming". He would like that students in the classroom received a recommendation of educational material on their smartphones during the class, in video format. The context information registered about this scenario is presented in Table 4 .

Table 4 defines the context A which will be located in classroom A and where there will be the course of "Algorithms and Programming," on Monday from 19:30 to 22:00. This information is previously registered in UbiGroup by the teacher using the web interface of CM agent. An example of this interface is shown in Figure 6.

Table 4. Example of context information

\begin{tabular}{|l|l|l|}
\hline \multicolumn{2}{|c|}{ Context A } \\
\hline \multirow{4}{*}{ Localization Properties } & NameIs & "classroom A" \\
\cline { 2 - 3 } & initGPSPosIs & $-29.792702,-51.152301$ \\
\cline { 2 - 3 } & endGPSPosIs & $0.00132,0.002642$ \\
\hline \multirow{5}{*}{ Activity Properties } & TypeIs & "Class" \\
\cline { 2 - 3 } & activityNameIs & "Alg. and Prog." \\
\cline { 2 - 3 } & descriptionIs & "Course of algorithms and programming - Prog. \\
& language Java" \\
\cline { 2 - 3 } Temporal Properties & KeywordIs & Class Standard Rule \\
\cline { 2 - 3 } & keywordIs & "Programming Language" \\
\hline \multirow{5}{*}{ Group Properties } & dayOfWeekIs & "Java" \\
\cline { 2 - 3 } & DateIs & - \\
\cline { 2 - 3 } & startTimeIs & $19: 30$ \\
\cline { 2 - 3 } & endTimeIs & $22: 00$ \\
\hline & groupTypeIs & Mix \\
\cline { 2 - 3 } & minLearnerNumberIs & 3 \\
\hline
\end{tabular}

Near the time of the beginning of class, students begin to arrive and are identified in context. To simplify the understanding of the scenario, we consider only profiles of the teacher and three students. Table 5 presents the information contained in the profiles. Profiles of students are maintained by the web interface of the PM agent as exemplified in Figure 7.

The $C M$ agent checks if the minimum number of learners was satisfied at each time a student enters the context. When the minimum is reached, the RE agent receives the learning context of the group of students, selects their profiles and identifies the most representative profile for the group with the assistance of $P M$ agent. In this scenario, student A was selected as the consensus profile (see Table 6).

Based on the recommendation rule contained in context (see Tables 2 and 3), the RE agent defines the filter parameters for LO metadata and sends this information to the $C O$ agent. The $\mathrm{CO}$ receives the search filters, creates a SPARQL query and sends the query to the LO repository. The $C O$ agent receives the results and forwards them to the RE agent. This agent sends the recommendation of content to the PS agent. This agent puts a notification on the top bar of the mobile device (see 
Figure 6. Web interface of $\mathrm{CM}$ agent

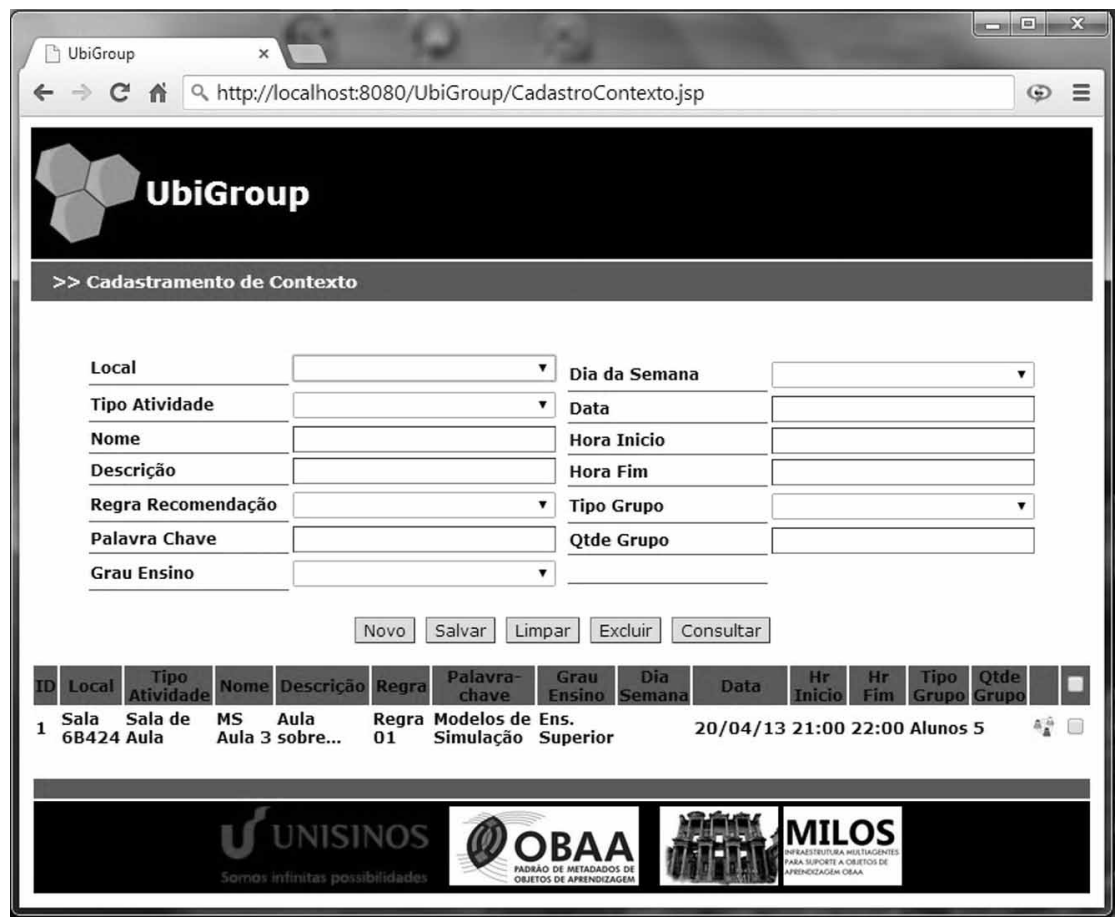

Figure. 8.a). When students select "View", the recommended materials are shown (see Figure 8.b). After viewing the LO, the students are invited to assess the recommendation, indicating whether they liked the material. These reviews are stored for use by the RE agent.

\section{CONCLUSION}

This article proposed UbiGroup, a model for ubiquitous recommendation of educational contents for groups of learners. UbiGroup recommends materials for dynamically managed groups which share a context. Furthermore, the model allows teachers to create their recommendation rules giving them freedom to align the search for materials to the teaching plan. Through the use of recommendation rules, it is possible to model the degree of refinement most appropriate for the pedagogical plan being implemented by the teacher.

The implementation of a prototype allowed the evaluation of the model by means of a scenario in a controlled environment. The results indicate that UbiGroup can recommend LO considering the context information and the most representative profile of the group. This is the case in the scenario presented in the article. The scenario also showed that the technology used by UbiGroup works and is ready for use in learning environments, except for a few details about the user interface, which will be discussed below.

The main contribution of UbiGroup is the recommendation for learner groups based on a consensus profile. Furthermore, the model detects the formation of dynamic groups and uses context information to guide the recommendation. In technological terms, the main differential of UbiGroup is the use of semantics in all phases of the recommendation process, including the detection of groups, handling of profiles and selection of LO contents. The semantics treatment is done by combining ontologies, metadata and agents' technologies. 
Table 5. Profiles used in the scenario

\begin{tabular}{|c|c|c|c|c|c|}
\hline \multicolumn{6}{|c|}{ Learning Profiles } \\
\hline \multirow{3}{*}{$\begin{array}{l}\text { Contact } \\
\text { Properties }\end{array}$} & learnerIDIs & 1 & 2 & 3 & 4 \\
\hline & nameIs & "Student A" & "Student B" & "Student C" & "Teacher D" \\
\hline & typeIs & "student" & "student" & "student" & "student" \\
\hline \multirow{6}{*}{$\begin{array}{l}\text { Portfolio } \\
\text { Properties }\end{array}$} & portfolioTypeIs & "paper" & "short-paper" & - & "paper" \\
\hline & titleIs & $\begin{array}{l}\text { "A multiagent } \\
\text { model for content } \\
\text { recommendation" }\end{array}$ & $\begin{array}{l}\text { "Recommendation of } \\
\text { educational contents } \\
\text { for groups" }\end{array}$ & - & $\begin{array}{l}\text { "A multiagent } \\
\text { model for content } \\
\text { recommendation", }\end{array}$ \\
\hline & descriptionIs & - & - & - & - \\
\hline & eventIs & "SBYY" & "ERYY" & - & "SBYY" \\
\hline & localIs & "City A" & "City B" & - & "City A" \\
\hline & yearIs & 2012 & 2012 & - & 2012 \\
\hline \multirow{6}{*}{$\begin{array}{l}\text { Grade } \\
\text { Properties }\end{array}$} & gradeTypeIs & "discipline" & "discipline" & "discipline" & - \\
\hline & gradeNameIs & "Alg. and Prog. I" & "Alg. and Prog. I" & "Alg and Prog. I" & - \\
\hline & descriptiveIs & - & - & - & - \\
\hline & institutionIs & "Inst. A" & "Inst. A" & "Inst. A" & - \\
\hline & evaluationIs & 7 & 8 & 8.3 & - \\
\hline & refDateIs & 2012-1 & $2012-1$ & $2012-2$ & - \\
\hline \multirow{4}{*}{$\begin{array}{l}\text { Preference } \\
\text { Properties }\end{array}$} & contentTypeIs & "video" & "video", "audio" & "video" & "video" \\
\hline & deviceTypeIs & "mobile" & "mobile" & "notebook" & "mobile" \\
\hline & visualIs & "visual" & "visual" & "visual" & - \\
\hline & prefContextIs & "classroom A" & "classroom A" & "classroom A" & "classroom A" \\
\hline
\end{tabular}

Figure 7. Web interface for the management of students' profiles

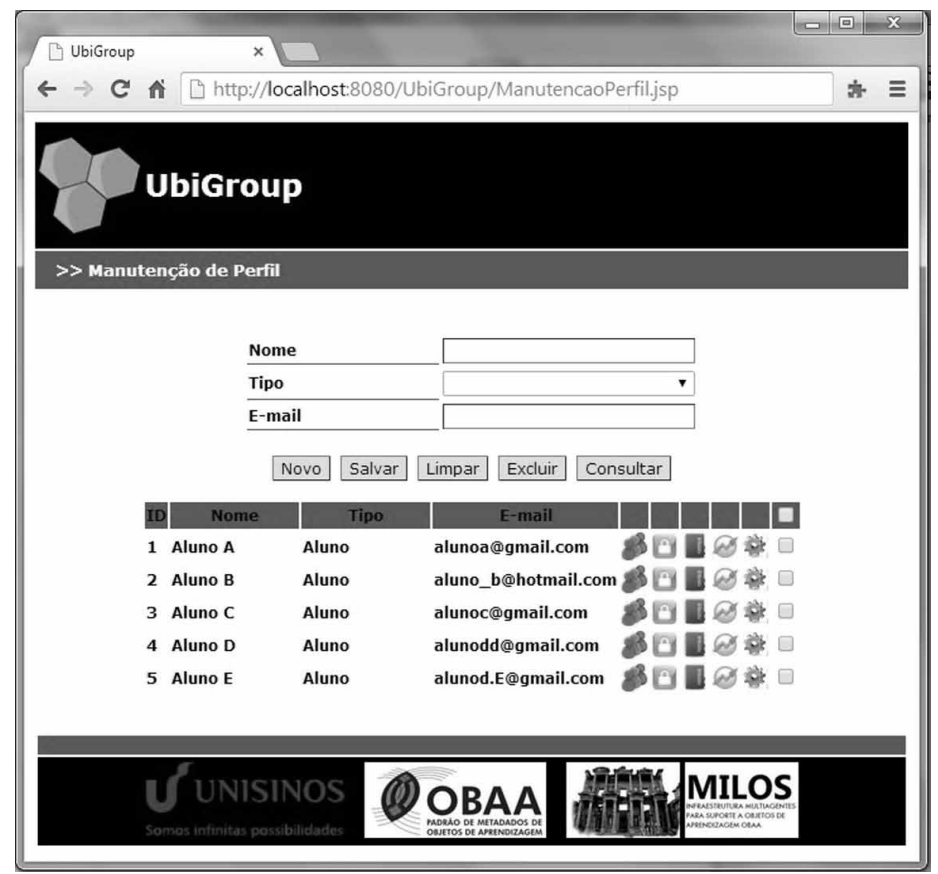


Table 6. Similarity of the profiles contained in the context

\begin{tabular}{|l|l|l|l|l|}
\hline & \multicolumn{1}{|c|}{ Student A } & \multicolumn{1}{c|}{ Student B } & \multicolumn{1}{c|}{ Student C } & \multicolumn{1}{c|}{ Teacher D } \\
\hline Student A & - & $67 \%$ & $47 \%$ & $53 \%$ \\
\hline Student B & $67 \%$ & - & $43 \%$ & $27 \%$ \\
\hline Student C & $47 \%$ & $47 \%$ & - & $7 \%$ \\
\hline Teacher D & $53 \%$ & $27 \%$ & $7 \%$ & - \\
\hline Avg. & $56 \%$ & $47 \%$ & $32 \%$ & $29 \%$ \\
\hline
\end{tabular}

Figure 8. Example of the mobile user interface

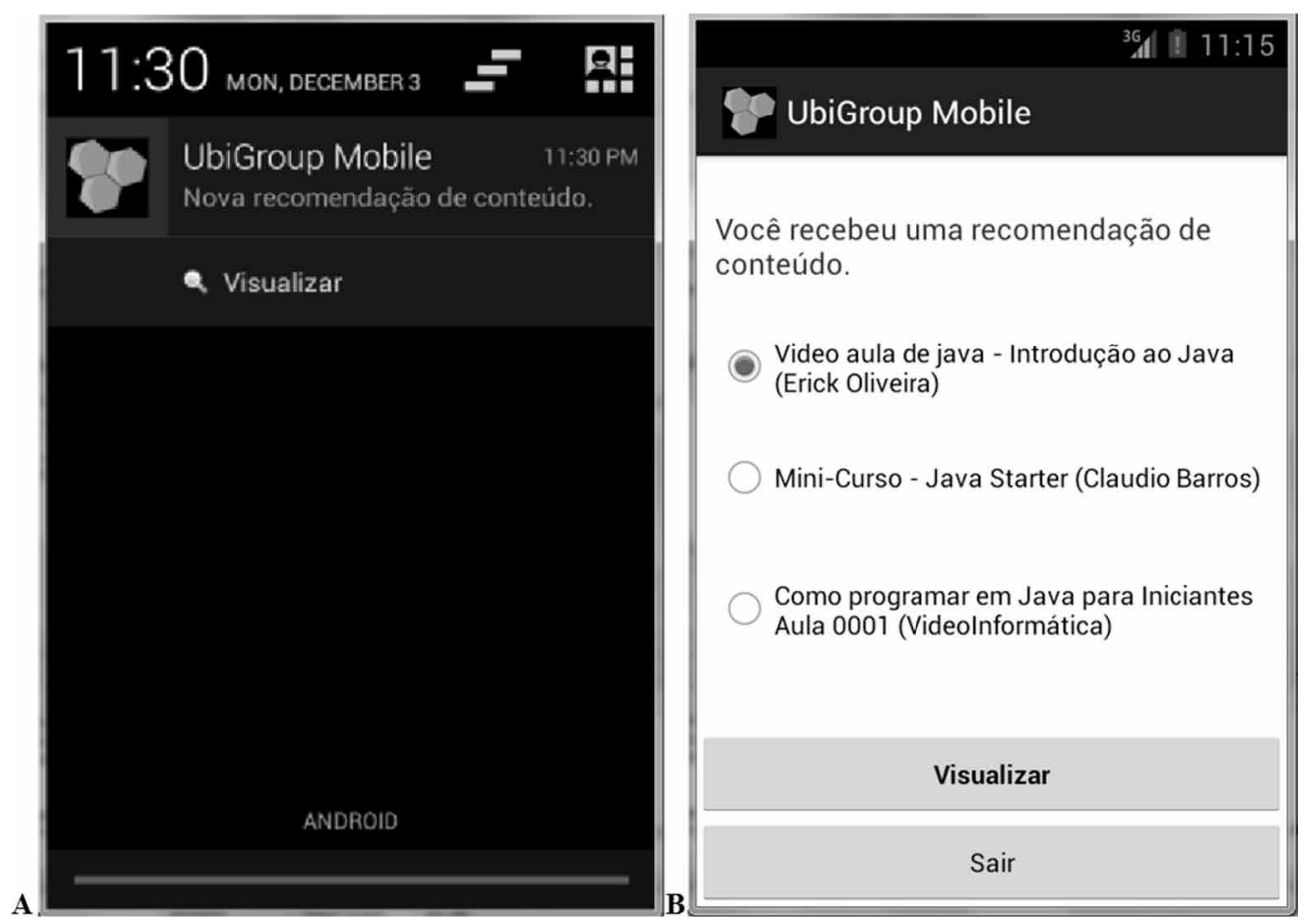

Currently, the main limiting factor of UbiGroup is the interface used by the teacher. This interface requires extensive and tedious specification of contexts and recommendation rules for the teaching plan used in the course. Future research will focus on the automation of these activities, based on ontological knowledge of the curriculum, learning domains, pedagogical strategies and teaching methods. This information can be combined with the physical (geographical) and social information about the institutions. We hope that with the use of these technologies, it will be possible to create a simple and useful interface for the teacher, automating the main activities of the generation of contexts and recommendation rules. In this sense, UbiGroup does not address the relationship between individual lessons. Teachers do not have the possibility to create dependencies between lessons or combinations to organize lessons sharing. We believe this is a relevant feature that can be added to UbiGroup, perhaps by including learnings objetcs chains and objects group recomendations following the chain. 
In addition, although the main focus of UbiGroup is in educational contexts, this is not a limiting factor of this model, because the technologies employed (ontologies, agents and metadata) can be applied to other domains related to information management. In future research, we intend to integrate UbiGroup with systems dedicated to competence management (Rosa et al., 2015), u-commerce (Barbosa et al., 2016), u-health (Pittoli et al., 2018) and u-accessibility (Barbosa et al., 2018). Currently, UbiGroup does not address data security aspects, mainly no treatment is done to separate public data (lessons) and private data (students' personal information). In this regard, future efforts will be directed to include security standards in the system. This feature will improve the educational application and allow better infrastructure to other applications. 


\section{REFERENCES}

W3C. (2012). W3C Recommendation: OWL 2 Web Ontology Language Structural Specification and FunctionalStyle Syntax (Second Edition). Retrieved from http://www.w3.org/TR/owl2-syntax

W3C. (2013). W3C Recommendation: SPARQL 1.1 Query Language. Retrieved from http://www.w3.org/TR/ sparql11-overview

Abech, M., Costa, C. A., Barbosa, J. L. V., Rigo, S. J., \& Righi, R. R. (2016). A model for learning objects adaptation in light of mobile and context-aware computing. Personal and Ubiquitous Computing, 20(2), 167-184. doi:10.1007/s00779-016-0902-3

Akbulut, Y., \& Cardak, C. S. (2012). Adaptive educational hypermedia accommodating learning styles: A content analysis of publications from 2000 to 2011. Computers \& Education, 58(2), 835-842. doi:10.1016/j. compedu.2011.10.008

Barbosa, J. L. V., Hahn, R. M., Barbosa, D. N. F., \& Saccol, A. I. D. C. Z. (2011). A ubiquitous learning model focused on learner interaction. International Journal of Learning Technology, 6(1), 62-83. doi:10.1504/ IJLT.2011.040150

Barbosa, J. L. V., Martins, C. J., Franco, L. K., \& Barbosa, D. N. F. (2016). TrailTrade: A model for trail-aware commerce support. Computers in Industry, 80, 43-53. doi:10.1016/j.compind.2016.04.006

Barbosa, J. L. V., Tavares, J., Cardoso, I., Mota, B., \& Martini, B. (2018). TrailCare: An Indoor and Outdoor Context-aware System to Assist Wheelchair Users. International Journal of Human-Computer Studies, 116, 1-14. doi:10.1016/j.ijhcs.2018.04.001

Bauer, B., \& Odell, J. (2005). UML 2.0 and agents: How to build agent-based systems with the new UML standard. Engineering Applications of Artificial Intelligence, 18(2), 141-157. doi:10.1016/j.engappai.2004.11.016

Boratto, L., Carta, S., \& Satta, M. (2010). Groups identification and individual recommendations in group recommendation algorithms. In Workshop on the Practical Use of Recommender Systems, Algorithms and Technologies (PRSAT) (pp. 27-34). Academic Press.

Christensen, I. A., \& Schiaffino, S. (2011). Entertainment recommender systems for group of users. Expert Systems with Applications, 38(11), 14127-14135.

Dey, A. K. (2001). Understanding and Using Context. Personal and Ubiquitous Computing, 5(1), 4-7. doi:10.1007/ s007790170019

Felder, R. M., \& Silverman, L. K. (1988). Learning and teaching styles in engineering education. Engineering education, 78(7), 674-681.

Ferreira, L. G. A. (2014). Ubigroup: An Ubiquitous Content Recommendation Model for Groups of Learners [Master's Degree Dissertation Thesis]. University of Vale do Rio dos Sinos (UNISINOS), Brazil. Retrieved from http://biblioteca.asav.org.br/vinculos/00000F/00000FEC.pdf

Giraffa, L., \& Vicari, R. (1998). The Use of Agents Techniques on Intelligent Tutoring Systems. In Proceedings of the 18th Int. Conf. of the Chilean Computer Science Society (pp. 76-83). IEEE. doi:10.1109/SCCC.1998.730785

Gluz, J. C., \& Silva, L. R. J. (2014). Ontological semantic search of learning objects: experiments and results. In Proceedings of the Brazilian Symposium on Computers and Education (SBIE) (pp. 837-846). Academic Press. doi:10.5753/cbie.sbie.2014.837

Gluz, J. C., Silva, L. R. J., \& Vicari, R. (2014). Aligning Ontologies to Bring Semantics to Learning Object Search. In Proceedings of the Int. Conf. on Intelligent Tutoring Systems (ITS) (pp. 619-620). Springer.

Gluz, J. C., \& Vicari, R. M. (2012). An OWL ontology for IEEE-LOM and OBAA metadata. In Proceedings of the Int. Conf. on Intelligent Tutoring Systems (ITS) (pp. 691-693). Springer.

Gluz, J. C., Vicari, R. M., \& Passerino, L. M. (2012). An Agent-Based Infrastructure for the Support of Learning Objects Life-Cycle. In Proceedings of the Int. Conf. on Intelligent Tutoring Systems (ITS) (pp. 696-698). Springer. doi:10.1007/978-3-642-30950-2_126 
Gomez-Perez, A., Fernández-López, M., \& Corcho-Garcia, O. (2004). Ontological engineering. Computer Review, 45(8), 478-479.

Guabassi, I. E., Bousalem, Z., \& Achhab, A. M. (2018). Personalized adaptive content system for context-aware ubiquitous learning. Procedia Computer Science, 127, 444-453. doi:10.1016/j.procs.2018.01.142

Guedes, G. T. A., \& Vicari, R. M. (2010). An UML Profile Oriented to the Requirements Modeling in Intelligent Tutoring Systems Projects. In Artificial Intelligence in Theory and Practice III (pp. 133-142). Springer.

Hightower, J. L., LaMarca, A. J., \& Smith, I. E. (2006). Practical Lessons from Place Lab. IEEE Pervasive Computing, 5(3), 32-39. doi:10.1109/MPRV.2006.55

IEEE-LTSC. (2002). Std 1484.12.1 IEEE Learning Technology Standard Committee (LTSC) Standard for Learning Object Metadata (LOM). Retrieved from http://standards.ieee.org/findstds/standard/1484.12.1-2002. html

Kardan, A. A., \& Ebrahimi, M. (2013). A novel approach to hybrid recommendation systems based on association rules mining for content recommendation in asynchronous discussion groups. Information Sciences, 219, 93-110. doi:10.1016/j.ins.2012.07.011

Kim, J. K., Kim, H. K., Oh, H. Y., \& Ryu, Y. U. (2010). A group recommendation system for online communities. International Journal of Information Management, 30(3), 212-219. doi:10.1016/j.ijinfomgt.2009.09.006

Klein, A. Z., Freitas, J. C. S., Balsasso, L., Barbosa, J. L. V., \& Mattiello, J. (2018). The Educational Affordances of Mobile Instant Messaging (MIM): Results of Whatsapp Used in Higher Education. International Journal of Distance Education Technologies, 16(2), 51-64. doi:10.4018/IJDET.2018040104

Kunze, J., \& Baker, T. (2007). The Dublin Core Metadata Element Set: RFC 5013. IETF. Retrieved from http:// www.ietf.org/rfc/rfc5013.txt

Levenshtein, V. I. (1966). Binary codes capable of correcting deletions, insertions, and reversals. Soviet Physics, Doklady, 10(8), 707-710.

Marinagi, C., Skourlas, C., \& Belsis, P. (2013). Employing ubiquitous computing devices and technologies in the higher education classroom of the future. Procedia: Social and Behavioral Sciences, 73, 487-494. doi:10.1016/j. sbspro.2013.02.081

PAPI. (2002). Standard for Learning Technology. Public and Private Information (PAPI) for Learners (PAPI learner). IEEE. Retrieved from http://metadata-standards.org/Document-library/Meeting-reports/ SC32WG2/2002-05-Seoul/WG2-SEL-044_SC36N0180_papi_learner_data_element_registry.pdf

Peterson, E. R., Rayner, S. G., \& Armstrong, S. J. (2009). Researching the psychology of cognitive style and learning style: Is there really a future? Learning and Individual Differences, 19(4), 518-523. doi:10.1016/j. lindif.2009.06.003

Pimmer, C., Mateescu, M., \& Gröhbiel, U. (2016). Mobile and ubiquitous learning in higher education settings. A systematic review of empirical studies. Computers in Human Behavior, 63, 490-501. doi:10.1016/j. chb.2016.05.057

Rosa, J. H., Barbosa, J. L. V., Kich, M., \& Brito, L. K. (2015). A Multi-Temporal Context-aware System for Competences Management. International Journal of Artificial Intelligence in Education, 1-38.

Russel, S. J., \& Norvig, P. (2010). Artificial Intelligence: A Modern Approach (3rd ed.). Prentice Hall.

Saccol, A. I. C. Z., Barbosa, J. L. V., Schlemmer, E., \& Reinhard, N. (2011). Mobile Learning in Organizations: Lessons Learned from Two Case Studies. International Journal of Information and Communication Technology Education, 7(3), 11-24. doi:10.4018/jicte.2011070102

Satyanarayanan, M. (2001). Pervasive computing: Vision and challenges. IEEE Personal Communications, 8(4), 10-17. doi:10.1109/98.943998

Tatar, D. E., Roschelle, J., Vahey, P., \& Penuel, W. R. (2003). Handhelds go to school: Lessons Learned. Computer, 36(9), 30-37. doi:10.1109/MC.2003.1231192 
Vianna, H. D., \& Barbosa, J. L. V. (2014). A Model for Ubiquitous Care of Noncommunicable Diseases. IEEE Journal of Biomedical and Health Informatics, 18(5), 1597-1606. doi:10.1109/JBHI.2013.2292860 PMID:25192571

Vicari, R. M., \& Gluz, J. C. (2007). An Intelligent Tutoring System (ITS) View on AOSE. International Journal of Agent-Oriented Software Engineering, 1(3-4), 295-333. doi:10.1504/IJAOSE.2007.016264

Vicari, R. M., Jaques, P., \& Verdin, R. (2008). Agent-Based Tutoring Systems by Cognitive and Affective Modeling. Hershey, PA; IGI Global. doi:10.4018/978-1-59904-768-3

Wagner, A., Barbosa, J. L. V., \& Barbosa, D. N. F. (2014). A model for profile management applied to ubiquitous learning environments. Expert Systems with Applications, 41(4), 2023-2034. doi:10.1016/j.eswa.2013.08.098

Webber, C. G., \& Lima, M. F. W. (2012). Evaluating automatic group formation mechanisms to promote collaborative learning - a case study. International Journal of Learning Technology, 7(3), 261-276. doi:10.1504/ IJLT.2012.049193

Weiser, M. (1991). The computer for the 21st century. Scientific American, 265(3), 94-104. doi:10.1038/ scientificamerican0991-94 PMID:1675486

Wiley, D. A. (Ed.). (2001). Connecting learning objects to instructional design theory: a definition, a metaphor, and a taxonomy. Utah State University. Retrieved from http://reusability.org/read/chapters/wiley.doc

Wooldridge, M. (2009). An introduction to multiagent systems. England: John Wiley and Sons, Ltd.

Zapata, A., Menéndez, V. H., Prieto, M. E., \& Romero, C. (2015). Evaluation and selection of group recommendation strategies for collaborative searching of learning objects. International Journal of HumanComputer Studies, 76, 22-39. doi:10.1016/j.ijhcs.2014.12.002

Luis Gustavo Araujo Ferreira received his BS degree in Computer Science from the La Salle University, Canoas, Brazil and MS degree in Applied Computing from the University of Vale do Rio dos Sinos (UNISINOS), São Leopoldo, Brazil. He has worked in the Software Management and also how Programmer and IT Analyst, and currently works as a PMO corporate at Bank Sicredi. Moreover, he is a collaborator in research projects at Mobilab.

Jorge Barbosa received his BS degree in Electrical Engineering from the Catholic University of Pelotas, Brazil, in 1991. He obtained his MS and PhD degrees in Computer Science from the Federal University of Rio Grande do Sul (UFRGS), Brazil, in 1996 and 2002, respectively. Nowadays, he is a full professor of Applied Computing Graduate Program (PPGCA) at the University of Vale do Rio dos Sinos (UNISINOS), São Leopoldo, Brazil. Additionally, he is a researcher of productivity at CNPq (the Brazilian Council for Scientific and Technological Development) and head of the Mobile Computing Laboratory (MobiLab/UNISINOS). His research interests include mobile and ubiquitous computing and mobile and ubiquitous learning. He is a member of the Brazilian Computer Society (SBC).

João Carlos Gluz has a PhD in Computer Science from Universidade Federal of Rio Grande do Sul (UFRGS), Brazil, and made postdoctoral studies in Assistive Technologies, Multimodal Interfaces and Accessibility Requirements at Universidad de Cordoba, Spain. He is professor and researcher in Software Engineering, Artificial Intelligence, Computing in Education, and Theory of Computation for twenty years. He also worked as software designer and $R \& D$ team leader of real-time embedded systems, like routers, remote access servers and network management systems for over ten years. He does joint research with Post-Graduation in Applied Computing (PPGCA) of UNISINOS and with Post-Graduation in Computing in Education (PGIE) of UFRGS. Currently he investigates forms to apply Artificial Intelligence and Virtual Reality to Education, and researches ways to evolve Artificial Intelligence and Software Engineering into a single integrated discipline.

Vitor Kehl Matter is a student at the University of Vale do Rio dos Sinos.

Debora Nice Ferrari Barbosa has a PhD. in Computer Science. Professor and researcher in Cultural Diversity and Social Inclusion. Additionally, she is a researcher of productivity at CNPq (the Brazilian Council for Scientific and Technological Development). Her research interests include ubiquitous learning systems, digital inclusion, game-based learning. She is a member of the Brazilian Computer Society (SBC). 